\title{
Diabetic Embryopathy
}

National Cancer Institute

\section{Source}

National Cancer Institute. Diabetic Embryopathy. NCI Thesaurus. Code C113485.

A condition in which the offspring of a mother with diabetes predating pregnancy

develops congenital malformations that can affect multiple organ systems including the brain and spinal cord, the heart and major vessels, the kidneys, the gut, and skeletal structures. 